\title{
Einführung zu den Hauptreferaten
}

Der Ophthalmologe sorgt und kämpft in seinem ärztlichen Handeln im allgemeinen fur die Erhaltung und Verbesse $\Gamma$ ung der spezifischen Sinnesfunktionen des Sehorganes. Die größte Bedrohung seiner Patienten besteht in der Erblindung. Aber ge-legentlich geht es doch noch um mehr; zu den lebensgefährlichen okularen Erkrankungen gehören in erster Linie die bösartigen Geschwülste des Auges und seiner Adnexe, vor allem der Lider und der Orbita. Es steht außer Frage, daß bei solchen Affektionen von vitaler Dignität, abgesehen von der rechtzeitigen Diagnose, die Wahl der richtigen Therapie und ihre zweckmäßigste Durch-führung von besonderer Bedeutung sein muß. Gerade aber weil in bezug auf die lndikation der verschiedenen Behandlungsprin-zipien - in erster Linie bei den Lidkarzinomen, aber auch bei manchen Typen epibulbärer, intraokularer und orbitaler Ge-schwulstbildungen - die Meinungen durchaus kompetenter Fachleute sehr stark auseinandergehen und der Einzelne, beson-ders der Praktiker, wegen der relativen Seltenheit dieser Affektionen oft nicht in der Lage ist, persönliche Erfahrungen zu sammeln, schien uns eine Darstellung der Indikationen, der Wege und Aussichten sowohl der Strahlentherapie wie der chirurgi-schen Behandlung dieser Tumoren von erheblichem praktischen Interesse.

Ich hoffe, Sie seien mit der Wahl unseres Hauptthemas einver-standen und freuen sieh mit uns, von berufener Seite vor allem einmal über die Möglichkeiten und Grenzen der aktinischen Therapie bei verschiedenen Geschwulstarten des Sehorgans und seiner näheren Umgebung orientiert zu werden.

Es ist uns eine besondere Freude, als ersten Referenten einen hervorragenden Vertreter einer Nachbardisziplin, der Dermato-logie, begrüßen zu dürfen. Wir wissen wohl alle um die großen maßgeblichen Erfahrungen Mieschers im schwierigen Felde der Histopathologie der Haut, vor allem der histogenetisch noch so ganz umstrittenen Hautkarzinome. Wir alle kennen seine eminen-ten Kenntnisse auf dem Gebiete der Strahlentherapie. Ich erinnere Sie nur an Mieschers eindrucksvolle Darstellung der Radium-

$17^{*}$

196

Einführung zu den Hauptreferate $\pi$

behandlung der Angiome, die vor wenigen Jahre $\pi$ in der Schwei-zerischen Medizinischen Wochenschrift erschienen ist.

Wie die einzelnen Spezialgebiete der Medizin nur in der Frei-heit der Beschränkung auf ihre besondere Domäne sich wirklich fortschreitend haben entwickeln können, so bedürfen sie heute wieder gegenseitiger Anlehnung und Anregung, urn vorwärtszu-kommen. Der Dermatologe wird nicht zögern, sich diagnostische und therapeutische Erfahrungen und Erkenntnisse der Ophthalmologie nutzbar zu machen, etwa hinsichtlich der Therapie des Lidekzems oder der syndermatotischen Katarakte. Auf derandern Seite soil der Augenarzt wissen, daß eine Behandlung der Lid-haut ohne Kenntnisse der Physiologie und Pathologie des wich-tigen «Organes Haut» selten geraten wird. So freuen wir uns auf die heutige, hoffentlich recht fruchtbare Synthese zwischen Der-matologie und Ophthalmologie. 
Au cours de Taprès-midi nous aurons le grand plaisir de pou-voir entendre un maître spécialiste dans un domaine de notre médecine de haute importance : le docteur Dollfus, de $\Gamma$ Hôpital de la Pitié, à Paris, nous parlera du traitement par rayons $\mathrm{X}$, des tumeurs épibulbaires, intra-oculaires et de Torbite. Voilà quelques mois seulement que Dollfus a fait à Tophtalmologie un cadeau utile et très précieux en son oeuvre magnifique sur le gliome de la rétine et les pseudogliomes, rapport présenté avec Auvers en 1953 à la Société française d'Ophtalmologie. Cette etude très complete s'occupe d'une façon étendue aussi de la thérapeutique du rétino-blastome, une tumeur qui, pour citer Dollfus, pose à Tophtal-mologiste un des problèmes des plus angoissants. Nous sommes heureux d'avoir parmi nous le docteur Dollfus comme représentant eminent de Tophtalmologie française à laquelle nous devons tant.

D. Den Abschluß der Referate bringt eine Darstellung der chirur-gisch-plastisch wiederherstellenden Therapie bei diesen verschie-denen Tumoren durch Herrn Stretff. Er ist durch jahrelange praktische Beschäftigung mit den sich hier stellenden biologischen und technischen Problemen dazu besonders berufen. Die Qualität unserer Referenten an deren Vorträge hoffentlich eine recht fruchtbare sachliche Diskussion sich anschließen wird bürgt wohl dafür

daß wir heute Interessantes zu hören bekommen werden. Möge davon vor allem auch zur Klärung unserer Meinungsbildung den Kranken recht viel zugute kommen.

F. Rintelen. 